\title{
Acute changes in Cerebrospinal Fluid 5-HIAA following Oral Paroxetine Challenge in Healthy Humans
}

\author{
Linda L Carpenter*,', George M Anderson ${ }^{2}$, Jason M Siniscalchi', Phillip B Chappell ${ }^{\mathbf{3}}$ and Lawrence H Price' \\ 'Butler Hospital, Laboratory for Clinical Neuroscience, Department of Psychiatry and Human Behavior, Brown Medical School, Providence, RI, \\ USA; ${ }^{2}$ Yale Child Study Center, Yale University School of Medicine, New Haven, CT, USA; ${ }^{3}$ Pfizer, Inc. Groton, CT, USA
}

\begin{abstract}
A number of studies have reported decreased human lumbar cerebrospinal fluid (CSF) concentrations of the major serotonin metabolite, 5-hydroxyindoleacetic acid (5-HIAA), following chronic administration of selective serotonin reuptake inhibitors (SSRIs). This decrease has been thought to be a consequence of elevated extracellular serotonin and to be mediated through terminal autoreceptor feedback inhibition of serotonin turnover. We wished to study the previously unexamined acute effects of SSRI administration on human CSF 5-HIAA. A serial lumbar puncture (LP) procedure was used to collect CSF samples before and after a single oral $40 \mathrm{mg}$ dose of the SSRI paroxetine (PAR) or matching placebo in eight healthy adult humans in a randomized, double-blind fashion. CSF 5-HIAA concentrations did not change following placebo, but showed a statistically significant $27 \%$ mean increase $3 \mathrm{~h}$ following PAR. Our findings stand in contrast to the decreases reported for CSF 5-HIAA after chronic SSRI treatment in humans and the decreases seen in brain extracellular 5-HIAA after acute or chronic administration of SSRIs to animals.
\end{abstract}

Neuropsychopharmacology (2003) 28, 339-347. doi:I 0. I038/sj.npp. I 300025

Keywords: cerebrospinal fluid (CSF); serotonin; 5-hydroxytryptamine (5-HT); 5-hydroxyindoleacetic acid (5-HIAA); paroxetine (PAR); selective serotonin reuptake inhibitor (SSRI)

\section{INTRODUCTION}

Selective serotonin reuptake inhibitors (SSRIs) are now widely used in the treatment of depression and anxiety disorders. The presumed mechanism of action, based on in vivo electrophysiological and intracerebral microdialysis studies in animals, is an enhanced serotonergic receptor stimulation caused by increased extracellular levels of serotonin (5-HT). Preclinical studies exploring changes in brain 5-hydroxytryptamine (5-HT) following systemic administration of various SSRIs have consistently found acute increases in extracellular fluid (ECF) 5-HT concentrations in cell body and terminal field regions of 5-HT neurons (Adell and Artigas, 1991; Bel and Artigas, 1992; Invernizzi et al, 1992). While the extent of the 5-HT increase has varied, increases have been reported in the raphe nuclei, hippocampus, hypothalamus, diencephalon, striatum, thalamus and frontal cortex (Fuller, 1994). In contrast, ECF levels of the major 5-HT metabolite, 5-hydroxyindoleacetic acid (5-HIAA), are consistently observed to decrease after acute SSRI administration. The decrease in ECF 5-HIAA has been attributed to somatodendritic and terminal autore-

\footnotetext{
*Correspondence: Dr Linda L Carpenter, Butler Hospital, Laboratory for Clinical Neuroscience, Department of Psychiatry and Human Behavior, Brown Medical School, 345 Blackstone Blvd, Providence, Rl 02906, USA. Tel: +I 401455 6349; Fax: + I 4014556534 , E-mail: linda_carpenter_md@brown.edu

Received 27 November 200 I; revised 19 June 2002; accepted I July 2002
}

ceptor-mediated reductions in the release and synthesis of 5-HT.

Some limited animal data are available regarding the chronic effects of the SSRIs on cerebraspinal fluid (CSF) or ECF levels of 5-HT or 5-HIAA (Bel and Artigas, 1993; Rutter et al, 1994; Invernizzi et al, 1996; Smith et al, 2000; Anderson et al, in press). There have also been a number of studies examining the effects of chronic SSRI treatment on human lumbar CSF 5-HIAA levels. These studies have consistently reported declines of CSF 5-HIAA of 40-60\% after weeks or months of SSRI administration (Bjerkenstedt et al, 1985; Potter et al, 1985; Scheinin, 1985; Martensson et al, 1991; De Bellis et al, 1993; Sheline et al, 1997). It is worth noting that the magnitude of the typical 5-HIAA decrease observed in human subjects is similar to that seen after acute SSRI administration in animals.

The absence of widely available techniques for assessing human ECF or obtaining multiple acute CSF samples has led to a lack of data regarding the acute effects of SSRIs in humans. One approach with the potential to obtain information on acute changes in CSF measures is that of continuous CSF sampling through an indwelling lumbar intrathecal catheter (Geracioti et al, 1992, 1993). This approach probably represents the closest currently available analogue to the in vivo microdialysis techniques used in animals. In the past several years, our research group has gained extensive experience using this method to study healthy human volunteers (Kirwin et al, 1997; Carpenter et al, 1998; Vythilingam et al, 2000). While this paradigm 
offers the unique opportunity to examine real-time dynamic changes in levels of CSF neurochemicals during pharmacological challenges, we found it to be associated with substantial adverse effects, generally in the form of postlumbar puncture (LP) headaches and other neurological sequelae, which frequently required treatment with intravenous fluids, sterile blood 'patch' procedures and prolonged bedrest.

The morbidity associated with the intrathecal lumbar catheter led us to consider other methods for serial CSF collection. The introduction of the Sprotte ${ }^{\mathbb{R}}$ spinal needle, which has a fine-caliber gauge and conical-shaped tip, has greatly enhanced subject comfort level and diminished adverse outcomes associated with LP (Sprotte et al, 1987; Pittoni et al, 1995). The greatly reduced incidence and severity of adverse effects suggested that multiple LP procedures would be feasible and might provide a practical alternative method for obtaining serial CSF samples.

We undertook the present study of healthy human subjects to (1) assess the feasibility of our serial LP method for detecting acute, pharmacologic probe-induced changes in relevant CNS neurochemicals in humans and (2) to test the hypothesis, generated from preclinical research findings, that a single oral dose of an SSRI would cause an acute decline in concentrations of CSF 5-HIAA.

In the context of the many methodological limitations that have faced investigators of human in vivo neurotransmitter function, measurement of plasma hormone concentrations after the acute administration of a challenge drug or other stimulus has become a widely used method for assessment of the effects of psychiatric illness on human neurotransmitter systems. Single doses of SSRIs are frequently used as pharmacological probes in neuroendocrine challenge tests of serotonergic function (eg Raap and Van de Kar, 1999; Attenburrow et al, 2001). Neuroendocrine challenge tests have been considered powerful tools for studying alterations in neural function. Acute changes in peripheral hormone concentrations following administration of a challenge drug have been interpreted as reflecting adaptive changes in receptor function in the brain which may hold value for predicting individual antidepressant response (Cowen, 1998). Pairing a serotonergic probe drug with specific 5-HT receptor antagonists in pharmacological challenge studies has become a widely used method for studying mechanisms of action of various antidepressants in both humans and animals. In animals, invasive procedures and direct examination of brain tissue and ECF allow for testing of basic assumptions about the effects of a pharmacological probe. Data describing relevant biological correlates of human neuroendocrine challenge tests, as measured in human CSF analytes or by brain imaging studies, may likewise provide collateral lines of evidence about the central effects of the probe drug in humans. An investigation involving contemporaneous examination of CSF and peripheral components (monoamines, neuropeptides and amino acids) during challenge tests provided the rationale for our implementation of continuous CSF sampling methods (Kirwin et al, 1997; Carpenter et al, 1998). A desire to couple our neuroendocrine challenge tests with a safer, more practical CSF sampling procedure provided the rationale for the present study.
Paroxetine (PAR) was selected for this study because, among currently available SSRIs, it has the highest affinity for the 5-HT transporter while exhibiting low affinity for other central neurotransmitter binding sites and receptors (Leonard 1992). PAR has no major metabolite and its absorption is not altered by a fast or fed state (Haddock et $a l, 1989)$. In addition, PAR is in wide clinical use, is welltolerated at a dose of $40 \mathrm{mg} / \mathrm{day}$, and has been reported to be an effective serotonergic neuroendocrine probe, with a single $40 \mathrm{mg}$ oral dose provoking a significant increase in serum cortisol $3 \mathrm{~h}$ after ingestion (Reist et al, 1996).

\section{SUBJECTS AND METHODS}

\section{Subjects}

The study was approved by the Institutional Review Board of Butler Hospital in Providence, RI. Eight healthy adult subjects $(5$ male, 3 female; mean \pm SD age, $28.6 \pm 10.8$ years; range, $19-51$ years), free of psychiatric or medical illness, gave voluntary, written informed consent to participate as paid volunteers in the study. Subjects were accepted if they had no personal or family history of medical, neurologic, or psychiatric illness, and no illicit drug use or regular medication use within the past year. All subjects were nonsmokers and demonstrated normal values on a battery of screening laboratory tests, which included serum chemistries, liver function tests, complete blood count, thyroid indices, urinalysis and urine toxicology screens, and pregnancy test in females. All subjects demonstrated normal results on physical examination and electrocardiogram before entering the study.

\section{Procedures}

Subjects participated in two separate but identical testing days, separated by at least 2 , but no more than 4 , weeks. Females were scheduled to participate in the follicular phase of their menstrual cycles. Subjects were advised to avoid strenuous physical activity for $24 \mathrm{~h}$ before, and for $48 \mathrm{~h}$ after, their participation in each testing day.

The protocol for serial CSF collection is outlined in Figure 1. After an overnight fast, subjects arrived at a dedicated biological testing suite at Butler Hospital and underwent placement of an intravenous catheter in a forearm vein for hydration and phlebotomy at $8 \mathrm{am}$. Usual caffeine intake (in the form of unsweetened coffee, tea or diet soda) was allowed, to avoid neurochemical perturbations that might be induced by acute caffeine withdrawal on the testing days. No other food or oral nutrition was given to subjects until after completion of the final LP. Hydration and adequate blood glucose levels were maintained via continuous intravenous fluid therapy with a solution of normal saline and $5 \%$ dextrose, at a rate of $150 \mathrm{ml} / \mathrm{h}$. At the end of the study $(6 \mathrm{pm})$, subjects resumed a regular diet and were fed dinner before being discharged home.

Bedrest began at $8 \mathrm{am}$, with subjects kept awake in a neutral testing environment throughout the day. Activity was limited to bedrest (except for bathroom use) until $1 \mathrm{~h}$ after the third LP. Reading materials and a television were made available for subjects to use throughout the day. Baseline self-report ratings of mood, anxiety and side effects 
Overnight fast, continue regular caffeine use to avoid withdrawal

$\begin{array}{ll}\text { 8:00 a.m. } & \text { Placement of intravenous line for hydration and phlebotomy } \\ & \text { Begin strict bedrest (awake in neutral environment) except bathroom } \\ & \text { Baseline ratings of mood and somatic effects } \\ \text { 8:45 a.m. } & \text { Pre-LP\#1 blood samples obtained } \\ \text { 9:00 a.m. } & \text { Lumbar Puncture \#1 (1 hour Pre-drug); CSF (6cc) collected } \\ \text { 9:15 a.m. } & \text { Post-LP \#1 blood samples obtained } \\ \text { 10:00 a.m. } & \text { Paroxetine } 40 \text { mg oral dose (or matching placebo) } \\ \text { 11:00 a.m. } & \text { Subject self-ratings of mood and somatic effects } \\ \text { 12:45 p.m. } & \text { Pre-LP\#2 blood samples obtained } \\ \text { 1:00 p.m. } & \text { Lumbar Puncture \#2 (3 Hours post-drug); CSF (6cc) collected } \\ \text { 1:15 p.m. } & \text { Post-LP \#2 blood samples obtained } \\ \text { 2:00 p.m. } & \text { Subject self-ratings of mood and somatic effects } \\ \text { 3:45 p.m. } & \text { Pre-LP\#3 blood samples obtained } \\ \text { 4:00 p.m. } & \text { Lumbar Puncture \#3 (6 Hours post-drug); CSF (6cc) collected } \\ \text { 4:15 p.m. } & \text { Post-LP \#3 blood samples obtained } \\ \text { 5:00 p.m. } & \text { Subject self-ratings of mood and somatic effects } \\ \text { 6:00 p.m. } & \text { Discontinue IV, dinner served, discharge subject home } \\ \text { Next day: phone contact to assess adverse effects (headache, lumbar soreness, etc) }\end{array}$

Figure I Serial CSF sampling protocol with fine-gauge Sprotte ${ }^{\mathbb{R}}$ spinal needle. Eight healthy subjects underwent two identical testing days with randomization to PAR ( $40 \mathrm{mg}$, single oral dose) or matching placebo.

were conducted at 8 am and again every $3 \mathrm{~h}$ until $5 \mathrm{pm}$ These included the Visual Analogue Scales (VAS) for 15 mood states (McCormack et al, 1988), an Adverse Symptoms Checklist (ASC; Rabkin et al, 1992), the Profile of Mood States (POMS; McNair et al, 1992), and the Patient-Rated Anxiety Scale (PRAS; Albus et al, 1990). Vital signs (heart rate, blood pressure, respiratory rate and oral temperature) were monitored at regular, frequent intervals throughout the testing day by a research clinician who was present in the room throughout the protocol.

Blood samples were obtained for cortisol assay through the existing intravenous line $5 \mathrm{~min}$ before and $5 \mathrm{~min}$ after each CSF collection. A total of $42 \mathrm{ml}$ of blood was withdrawn during the course of each testing day. Cortisol levels were measured to confirm the neuroendocrine 'signal' of PAR challenge (change in resting cortisol from 9 am to $1 \mathrm{pm}$ on PAR vs placebo days) as well as to provide a measurement of 'stress' (presumed HPA arousal from emotional or somatosensory input) related to the LP procedures themselves (pre-LP vs post-LP values at each time point on both days).

For each of the three LP procedures ( $9 \mathrm{am}, 1 \mathrm{pm}$ and 4 $\mathrm{pm})$, subjects were placed in a comfortable sitting upright position (arms and head leaning on a tray table) on the edge of the hospital bed. Standard sterile preparation and intradermal lidocaine $1 \%$ anesthesia were applied at the L3-L4 or L4-L5 interspace (with all CSF samples from any given subject drawn from the same anatomical location on both test days). A 20-gauge introducer needle was inserted to penetrate the skin and superficial tissues, followed by insertion of the Sprotte ${ }^{\mathbb{R}}$ 25-gauge pencil-point spinal needle to the subarachnoid space. At each LP, $6 \mathrm{ml}$ of spinal fluid were removed, subsequently divided into $0.5 \mathrm{ml}$ aliquots, and frozen at $-80^{\circ} \mathrm{C}$ until assayed. Potential circadian rhythm effects on CSF production and clearance were controlled by conducting the LPs at exactly the same time points on both testing days. Following each LP procedure, subjects were returned to bedrest in the recumbent position. Subjects underwent double-blind randomization to receive either PAR $40 \mathrm{mg}$ or matching placebo in a single oral dose at 10 am Potential circadian rhythm effects on CSF production and clearance were controlled by conducting the LPs at exactly the same time points on both testing days.

Following a final neurological evaluation and dinner at 6:00 pm, subjects were discharged home with instructions to contact a physician investigator in the event of emergent headache or other adverse outcome. Subjects were routinely contacted by phone the following day to complete an assessment of possible adverse effects related to the procedure. Ibuprofen was recommended on an as-needed basis for mild tenderness at the puncture site.

\section{Hormone and Amino Acid Assays}

Levels of CSF tryptophan (TRP), tyrosine (TYR), 5-HIAA, and homovanillic acid (HVA) were determined using a 
modified version of a previously described high performance liquid chromatographic (HPLC) method (Anderson et al, 1979; Leckman et al, 1988). Intra- and interassay coefficients of variation (CVs) were between 5 and $11 \%$. The concentration of CSF norepinephrine (NE) was determined by HPLC with aperometric detection after alumina extraction of $0.5 \mathrm{ml}$ of CSF; the interassay CV of this method is 7.5\% (Anderson et al, 1988; Leckman et al, 1995). Plasma cortisol concentrations were determined using the GammaCoat cortisol I-125 coated-tube radioimmunoassay (RIA) kit (INCSTAR Corp., Stillwater, MI). The intra- and interassay CVs observed for quality assessment samples (5 and $20 \mathrm{ug} / \mathrm{dl}$ ) were less than 5 and $10 \%$, respectively.

\section{Data Analysis}

Concentrations of CSF TRP, TYR, HVA, NE, and 5-HIAA, pre-LP plasma cortisol, post-LP plasma cortisol, and pre- $v s$ post-LP change in cortisol (LP $\Delta$ CORT) were analyzed for change over time ( 3 time points), drug (PAR vs placebo) effects, and time $\times$ drug interactions using a repeated measures factorial design analysis of variance (ANOVA). Where sphericity assumptions were not met, the HuyhnFeldt correction was applied. Post hoc $t$-tests were computed when significant main effects were detected. Additionally, $t$ tests were applied to compare the baseline (9 am) and 3-h postdrug $(1 \mathrm{pm})$ concentrations, since peak drug effects were expected to be evident at that time point. Change in concentration from baseline to $1 \mathrm{pm}$ was calculated for each individual and described here as ' $\Delta 9-1$ '. Paired $t$-tests were used to compare pre- and post-LP plasma cortisol concentrations for each of the three LP procedures. Alpha level was set at 0.05 for determination of significance in all analyses of neurochemical data. Mood, side effects and physiological data were analyzed for change over time and drug effects with repeated measures ANOVA and post hoc $t$ tests where indicated. These analyses included the PRAS total score, 15 VAS ratings, 9 POMS factor scores describing mood states, and any adverse effects items endorsed as present by more than one subject during the protocol. The VAS scales were: 'angry,' 'anxious,' 'calm,' 'depressed,' 'drowsy,' 'energetic,' 'fearful,' 'happy,' 'high,' 'hungry,' 'irritable,' 'mellow,' 'nervous,' 'sad,' 'talkative.' The six original POMS factor scores (tension/anxiety, depression/ dejection, anger/hostility, fatigue/inertia, vigor/activity, confusion/bewilderment) were supplemented with three additional POMS factor scores (elation, arousal, positive mood; Fischman et al, 1990). Only ten adverse effects items on the checklist were endorsed by one or more subjects. These were: drowsy/sleepy, irritable, trouble concentrating, headache, dry mouth, muscle cramps/stiffness, trouble sitting still, excessive sweating, appetite increase and weakness/fatigue. The Bonferonni correction was applied to control for multiple comparisons, resulting in a corrected alpha of 0.001 for significance on these measures.

\section{RESULTS}

\section{CSF Neurochemical Effects of PAR}

Mean CSF concentrations of NE, TRP, TYR, HVA and 5HIAA are plotted over time for both test days in Figure 2.
Consistent with what might be expected in a fasting state throughout the day, significant linear decreases in CSF concentrations of both TRP and TYR were seen over time $(\mathrm{F}[1.3,17]=24.00, p<0.001$ and $\mathrm{F}[2,26]=33.57, p<0.001$, respectively). This pattern appeared to be unaffected by active drug. There were no significant time or drug effects on CSF concentration of NE.

A significant time effect was seen in CSF concentrations of 5-HIAA $(\mathrm{F}[2,26]=3.48, p<0.05)$, attributable to the difference between the PAR and placebo groups at $1 \mathrm{pm}$ (Figures 2 and 3). Specifically, active PAR significantly increased CSF 5-HIAA at $3 \mathrm{~h}$ after drug ingestion (from baseline mean $11.9 \pm 1.7 \mathrm{ng} / \mathrm{ml}$ to post-PAR mean $15.1 \pm 3.5 \mathrm{ng} / \mathrm{ml}, t=3.41, p=0.01$ ), while administration of placebo had no significant effect on CSF 5-HIAA levels (baseline mean $11.2 \pm 2.4 \mathrm{ng} / \mathrm{ml}$ to post-placebo mean $11.9 \pm 2.8 \mathrm{ng} / \mathrm{ml}, \quad t=0.78, \quad p=0.462$; cf. Figure 3$). \quad$ A significant acute rise (mean \pm SD, $27 \pm 21 \%$ ) in CSF 5HIAA was attributable to active PAR, as reflected in a significantly greater $\Delta 9-1$ on the active drug day $(t=2.79$, $p=0.027)$. Group differences in CSF 5-HIAA did not persist at the 6-h post-drug timepoint (4 pm).

Repeated measures ANOVA did not show significant time or drug effects for CSF HVA, but secondary analyses revealed that the pre- to post-drug change $(\Delta 9-1)$ in CSF concentration of HVA was significantly greater in the active PAR group than in the placebo group $(6.41 \pm 6.67 \mathrm{ng} / \mathrm{ml}$ for PAR vs $0.99 \pm 7.18 \mathrm{ng} / \mathrm{ml}$ for placebo, $t=2.45, p=0.05$ ), reflecting a mean $31 \pm 36 \%$ PAR-induced increase in CSF HVA $3 \mathrm{~h}$ after drug ingestion. Consistent with patterns observed by other researchers (Jibson et al, 1990), we found CSF HVA levels highly correlated with CSF 5-HIAA levels in both the placebo $(r=0.64, p=0.001)$ and active drug $(r=0.75, p<0.001)$ conditions.

\section{Plasma Cortisol Effects of LP Stress and PAR}

Pre- and post-LP plasma cortisol concentrations are presented graphically for both the placebo and PAR test days in Figure 4. Pre-LP samples were considered representative of 'resting state' or 'basal' cortisol levels, while post-LP samples were thought to reflect a 'stress-induced state,' with acute activation of the hypothalamus-pituitaryadrenal (HPA) axis by the emotional and somatic experience of the LP procedure itself. Comparison of pre- $v s$ postLP cortisol levels was used to grossly reflect the degree of HPA-axis activation caused by LP stress.

Consistent with known patterns of diurnal variation, unprovoked cortisol levels (pre-LP samples) were generally highest in the morning and lower in the afternoon $(\mathrm{F}[2,28]=23.1, p<0.001)$, and active drug did not significantly influence this pattern. A neuroendocrine 'signal' was confirmed by a significantly different pattern of resting cortisol (pre-LP samples) change from baseline (9 am) to 1 $\mathrm{pm}$ ( $3 \mathrm{~h}$ after challenge drug) between the active drug and placebo days, reflecting a relative increase in cortisol attributable to PAR $(t=-2.9 ; p=0.02)$.

Significant LP-induced increases in cortisol were not observed for the 9 am or $4 \mathrm{pm}$ procedures on either placebo or active PAR days. However, the $1 \mathrm{pm} \mathrm{LP}$ procedure produced a trend-level cortisol increase on the placebo day $(8.65 \pm 3.45 \mu \mathrm{g} / \mathrm{dl}$ pre-LP $v s 14.60 \pm 7.61 \mu \mathrm{g} / \mathrm{dl}$

Neuropsychopharmacology 
$\mathrm{NE}(\mathrm{pg} / \mathrm{ml})$

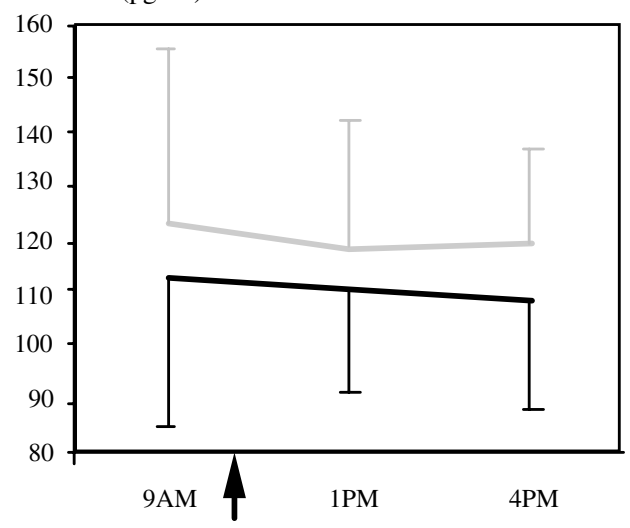

$\operatorname{TRP}(\mathrm{pg} / \mathrm{ml})$
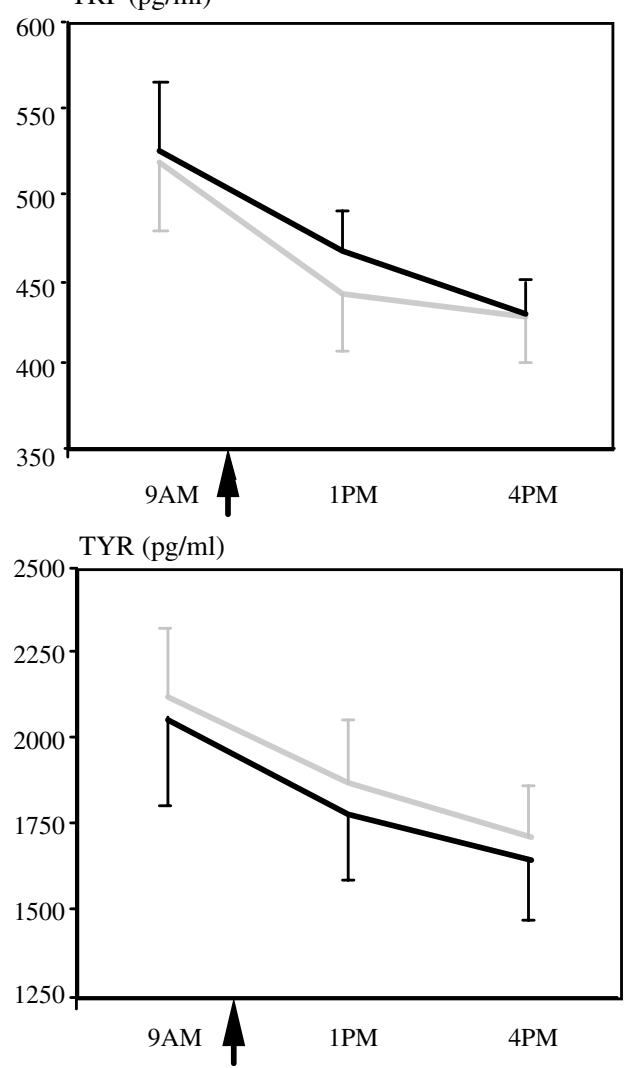

HVA $(\mathrm{pg} / \mathrm{ml})$

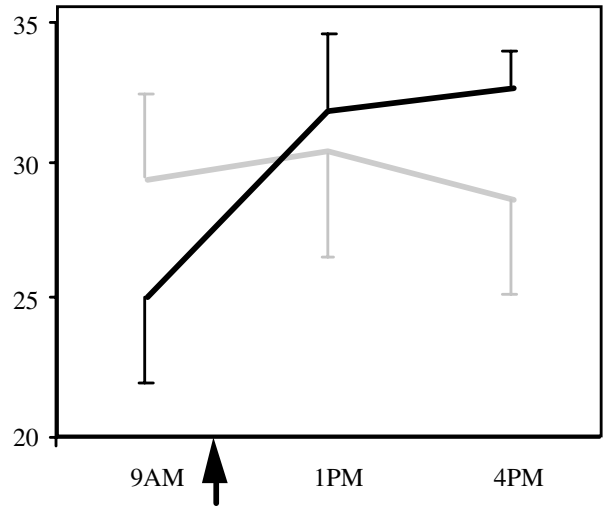

5-HIAA $(\mathrm{ng} / \mathrm{ml})$

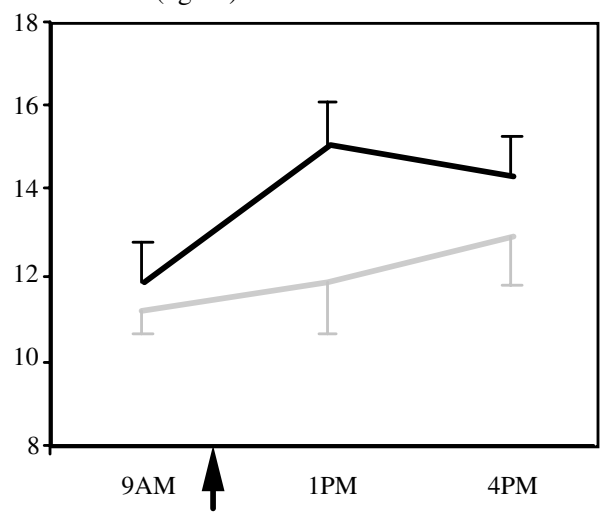

Figure 2 CSF mean concentrations (with standard deviation bars) plotted over 3 time points for norepinephrine (NE), homovanillic acid (HVA), tryptophan (TRP), 5-hydroxyindolacetic acid (5-HIAA), and tyrosine (TYR). Arrow indicates oral administration of single oral dose PAR 40 mg (dark line) or matching placebo (light line) or at 10:00 am.

post-LP, $t=2.05, p=0.08)$ and a statistically significant cortisol increase on the PAR day $(10.03 \pm 2.08 \mu \mathrm{g} / \mathrm{dl}$ pre-LP vs $17.30 \pm 5.05 \mu \mathrm{g} / \mathrm{dl}$ post-LP, $t=4.53, p=0.003)$. Since all three LP procedures were conducted in an identical manner, this finding can be interpreted as a PAR-enhanced HPA response to the stress of the LP procedure.

\section{Subjective and Physiological Effects of PAR}

No time, drug or interaction effects were found on the PRAS total score for state anxiety. Increases in VAS ratings of 'hunger,' 'mellow' and 'talkative' over time, and decreases in ratings of 'nervous' over time, were noted before application of the Bonferonni correction. However, after controlling for multiple comparisons, time effects were found on only one of the 15 VAS items, 'hunger,' which increased throughout the day $(\mathrm{F}[3,42]=20.3, p<0.001)$ as patients were fasting during the protocol. A time $\times$ group interaction reflected lower scores on ratings of 'calm' in the active PAR group $4 \mathrm{~h}$ after drug ingestion $(\mathrm{F}[2.5,42]=3.6, p=0.03)$, but this finding was lost when the more stringent alpha criterion was applied. Analysis of mood data from the POMS revealed trends toward general decreases in 'elation' $(\mathrm{F}[3,30]=2.9$, $p=0.05)$ and 'vigor' $(\mathrm{F}[3,30]=4.8, p=0.006)$ over time, 


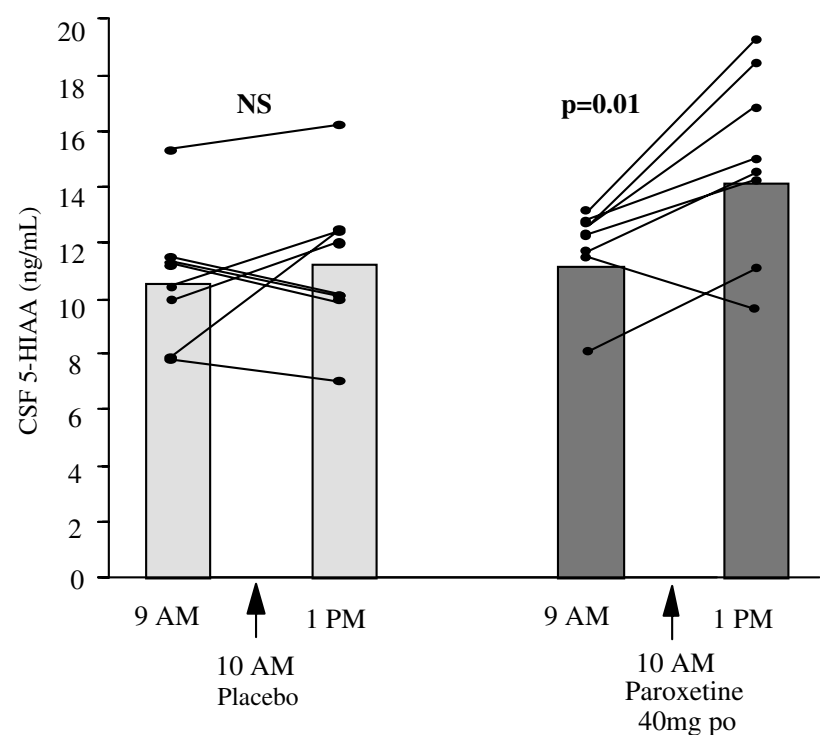

Figure 3 CSF 5-HIAA concentrations before and after PAR or placebo challenge. Raw data are plotted over bars indicating mean values of CSF 5HIAA concentrations. Paired t-tests were used to compare the baseline and postdrug values: placebo condition (baseline mean 11.2, postdrug mean I I.9) $t=2.79, p=0.462$; active PAR (baseline mean I I.9, postdrug mean I5.I) $t=3.4 \mid, p=0.01 \mathrm{I}$.

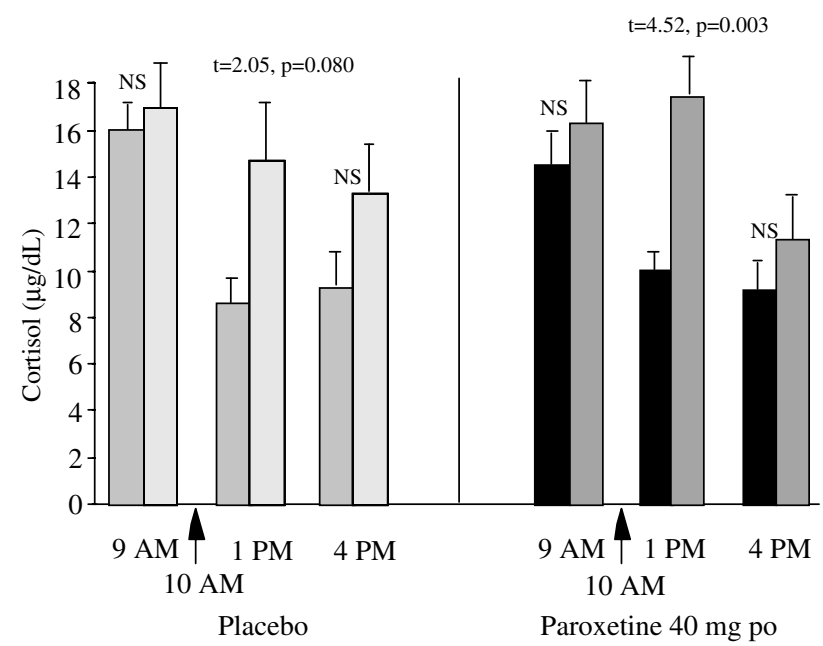

Figure 4 Plasma cortisol levels during PAR or placebo challenge. Bars represent mean (+SE) plasma cortisol levels for eight healthy control subjects during each of two testing days. Plasma samples were drawn through existing intravenous catheter line immediately before, and immediately following, each LP. t-Tests indicate the comparison of preand post-LP cortisol concentrations at each time point.

but there were no statistically significant effects found after Bonferonni correction.

No significant time, group or interaction effects emerged in the side effects data. Physiological measures revealed no significant changes over time or by group. However, a trend was noted for an overall time effect in systolic blood pressure (lower in the morning and higher in the afternoon, $\mathrm{F}[3,42]=2.5, p=0.07$ ); post hoc tests identified this trend as attributable to active PAR $(\mathrm{F}[3,21]=2.65, p=0.07)$.
None of the subjects experienced post-LP headache/ neurological symptoms or required blood-patch procedures. All subjects completed the study by returning for the second test day.

\section{DISCUSSION}

The present investigation demonstrated a significant mean $27 \%$ rise in concentrations of the 5-HT metabolite 5-HIAA in lumbar CSF $3 \mathrm{~h}$ after administration of a single $40-\mathrm{mg}$ dose of oral PAR in eight healthy humans. This major finding is in opposition to the well-established observation of decreased 5-HIAA in human lumbar CSF after chronic SSRI treatment. It is also at variance with the many preclinical observations of decreased brain ECF levels of 5HIAA after acute administration of SSRIs. Several factors deserve consideration in attempting to account for these unexpected findings.

The discrepancy with previous observations in humans receiving chronically administered SSRIs may be because of differing acute and chronic effects of SSRIs in humans. The consistent and similar decreases observed in ECF 5-HIAA in acute animal studies and in lumbar CSF 5-HIAA in chronic treatment human studies would seem to argue against this possibility. Moreover, recent studies of 5-HT and 5-HIAA in monkey brain ECF and cisternal CSF fail to support the existence of major differences across species. These studies have shown that central 5-HT release in monkeys increases after acute (Smith et al, 2000) or sustained (Anderson et al, in press) SSRI administration. The same studies have observed marked declines in ECF or cisternal CSF 5-HIAA after acute or chronic SSRI administration. Thus, the nonhuman primate data appear consistent with the previous rodent and human data in finding acute increases in 5-HT release and acute and chronic decreases in 5-HIAA.

It must also be noted that human lumbar CSF 5-HIAA levels reflect in large part the turnover of 5-HT in spinal serotonergic neurons. These neurons may have a substantially different acute response to an SSRI than the rostrally projecting neurons that are assessed in animal brain microdialysis experiments. Limited support for this possibility is provided by microdialysis studies that have reported regional differences in the acute effects of acute SSRIs on 5-HT release (Fuller, 1994; Kreiss and Lucki, 1994).

Another possible explanation for the present findings is suggested by a report of increased ECF 5-HIAA in the rat after restraint or immobilization stress (Lucki, 1997). Increases in striatal and hippocampal ECF 5-HIAA of about $30-60 \%$ were observed $2-3 \mathrm{~h}$ after the start of $90 \mathrm{~min}$ of restraint stress. While no definite explanation was offered for the 5-HIAA increase (which occurred in the absence of significant change in ECF 5-HT), it was suggested that reported increases in brain tryptophan after immobilization could have provided a physiological basis for the elevation (Lucki, 1997). However, CSF tryptophan actually declined in our study, apparently eliminating this as a explanation for the increased CSF 5-HIAA seen here. Moreover, since our data were collected using a within-subjects, placebo-controlled design, it is unlikely that the PAR- 
induced increase in CSF 5-HIAA can be attributable to the stress of the LP procedure itself.

Peripheral cortisol measurements were used to confirm the neuroendocrine 'signal' of PAR, and also as an indication of stress attributable to the LP procedures. Statistically significant LP-induced increases in cortisol did not occur during the 9 am and 4 pm procedures, even though the diurnal pattern of cortisol release would favor detection of such a stress effect in the late afternoon (i.e 4 $\mathrm{pm})$. At the mid-day $(1 \mathrm{pm})$ procedure, a trend-level rise in cortisol was noted on the placebo day, and a significant LPinduced cortisol increase was seen on the PAR day. Vital signs and self-ratings of anxiety/mood state did not support the hypothesis that the $1 \mathrm{pm}$ LP was more emotionally disturbing or physiologically arousing than the same procedure performed at the other time points.

Even in the absence of significant physical or psychological stress, however, it is possible that the stress-like neuroendocrine effects of acute PAR administration (eg activation of hypothalamus) may have influenced the observed neurochemical response. Although such neuroendocrine effects have been reported to vary in intensity between specific SSRIs and across studies (Calogero et al, 1990; Fuller, 1992; Reist et al, 1996; Attenburrow et al, 2001), acutely administered SSRIs are generally thought to increase the release of pituitary hormones, including prolactin and adrenocorticotrophic hormone (ACTH). Consistent with this, an enhancing effect of PAR on LP-induced cortisol secretion was apparent in our subjects.

Other investigators (McEwen, 1987; Chaouloff, 1993) have observed that various stressful conditions appear to exert permissive effects on 5-HT biosynthesis and dampening effects on neuronal sensitivity to 5-HT, probably mediated by HPA-axis activation. In the present context, direct activation of the HPA axis might have resulted in attenuation of the normally occurring autoreceptor feedback inhibition of 5-HT release and synthesis, resulting in the observed increase in CSF 5-HIAA.

Pharmacokinetic factors might have played a role if drug levels and the extent of transporter blockade were decreasing several hours after PAR administration. In such a situation, quite different from that present in chronic studies, compensatory increases in 5-HT metabolism might have occurred in an attempt to overcome the initial autoreceptor-mediated reduction in 5-HT release and synthesis. However, given the relatively long half-life $(20 \mathrm{~h})$ of PAR (Haddock et al, 1989), it is unlikely that substantial decreases in reuptake inhibition occurred before the $1 \mathrm{pm}$ or $4 \mathrm{pm}$ CSF samples were obtained. We did not measure CSF or plasma PAR concentrations, or other peripheral indices of 5-HT function that would have provided additional information about the possible contribution of relevant pharmacokinetic variables. The present investigation did not assess CSF neurochemical changes associated with efficacious or therapeutic doses of PAR, but rather was intended to shed light on the acute effects of an SSRI on central 5-HT activity in humans and provide CSF correlates for the peripheral hormone changes which occur during SSRI challenge tests.

Finally, the relationship between 5-HIAA and the dopamine metabolite, HVA, may be informative. A high correlation between the two CSF metabolites is frequently observed (Faull et al, 1981; Risby et al, 1987), with results from several studies suggesting that loss of this relationship is predictive of nonresponse to antidepressant treatment (Hsiao et al, 1987; Risby et al, 1987). While not clearly understood, the origin of the monoamine metabolite intercorrelation in human lumbar CSF does not appear to be mainly attributable to competition for the shared probenecid-sensitive organic acid transport system (Jibson et al, 1990), and may reflect functional interactions between the dopaminergic and 5-HT systems. Although some of the post-SSRI rise in CSF HVA we observed may be because of the rise in (and increased competition from) 5-HIAA, but the data also raise the possibility that PAR may be affecting, directly or indirectly, the activity of the acid transport system.

With regard to feasibility, the serial LP sampling method we used was easily executed and appeared to induce minimal HPA arousal, except when coupled with active PAR. While no invasive procedure could be construed as totally 'stress-free', we found the testing days to be very well tolerated by our subjects, each of whom underwent two test days with 3 LPs each day. Most subjects complained of boredom with prolonged bedrest, but described the CSF sampling procedure itself as less painful than the intravenous catheter insertion. None experienced headache or significant adverse outcomes, leading us to conclude that fine-gauge, conical-tip SPROTTE ${ }^{\circledR}$ needles allow for serial CSF sampling and meaningful assessment of central neurochemical dynamics in humans with minimal discomfort and morbidity.

Interpretation of intriguing findings in the setting of new methodology warrants considerable caution, leading to the speculative nature of our discussion. The surprising observation of acute increases in human lumbar CSF 5HIAA after SSRI administration indicates that additional animal studies are warranted. Especially relevant would be studies in nonhuman primates examining the short- and long-term effects of SSRIs on lumbar CSF levels of 5-HIAA and on cisternal CSF 5-HT and 5-HIAA levels. Clinical studies exploring possible group differences in CSF 5-HIAA response to SSRI across control and patient groups would also be of interest. Functional brain imaging data may also provide useful correlates for peripheral and CSF findings in neuroendocrine challenge tests. Examination of possible relationships between neurochemical changes and treatment response may provide useful information and approaches regarding outcome prediction and treatment selection. Taken together, research in this area may help clarify the mechanism of action of the SSRIs and shed light on the pathophysiology of depression.

\section{ACKNOWLEDGEMENTS}

This work was supported in part by a NARSAD Young Investigator Award (Dr Carpenter), NIMH grant MH 30929, and by a grant from Pfizer, Inc. Spinal needles were donated by B Braun Medical, Inc. Laura Hall, MS assisted in laboratory assay procedures, and Sarah Yasmin, MD assisted in clinical administration of the protocol. 


\section{REFERENCES}

Adell A, Artigas F (1991). Differential effects of clomipramine given locally or systemically on extracellular 5-hydroxytryptamine in raphe nuclei and frontal cortex. An in vivo brain microdialysis study. Naunyn Schmiedebergs Arch Pharmacol 343: 237-244.

Albus M, Maier W, Shera D, Bech P (1990). Consistencies and discrepancies in self- and observer-rated anxiety scales. A comparison between the self- and observer-rated MarksSheehan scales. Eur Arch Psychiatry Clin Neurosci 240: 96-102.

Anderson GM, Bennett AJ, Weld KP, Pushkas JG, Ocame DM, Higley JD (2002). Serotonin in cerebrospinal fluid of rhesus monkeys: basal levels and effects of sertraline administration. Psychopharmacology 161: 95-99.

Anderson GM, Durkin TA, Chakraborty M, Cohen DJ (1988). Liquid chromatographic determination of taurine in whole blood, plasma and platelets. J Chromatogr 431: 400-405.

Anderson GM, Young JG, Cohen DJ (1979). Rapid liquid chromatographic determination of tryptophan, tyrosine, 5hydroxyindoleacetic acid and homovanillic acid in cerebrospinal fluid. J Chromatogr 164: 501-505.

Attenburrow MJ, Mitter PR, Whale R, Terao T, Cowen PJ (2001). Low-dose citalopram as a 5-HT neuroendocrine probe. Psychopharmacology (Berl) 155: 323-326.

Bel N, Artigas F (1992). Fluvoxamine preferentially increases extracellular 5-hydroxytryptamine in the raphe nuclei: an in vivo microdialysis study. Eur J Pharmacol 229: 101-103.

Bel N, Artigas F (1993). Chronic treatment with fluvoxamine increases extracellular serotonin in frontal cortex but not in raphe nuclei. Synapse 15: 243-245.

Bjerkenstedt L, Edman G, Hagenfeldt L, Sedvall G, Wiesel FA (1985). Plasma amino acids in relation to cerebrospinal fluid monoamine metabolites in schizophrenic patients and healthy controls. Br J Psychiatry 147: 276-282.

Calogero AE, Bagdy G, Szemeredi K, Tartaglia ME, Gold PW, Chrousos GP (1990). Mechanisms of serotonin receptor agonistinduced activation of the hypothalamic-pituitary-adrenal axis in the rat. Endocrinology 126: 1888-1894.

Carpenter LL, Anderson GM, Pelton GH, Gudin JA, Kirwin PD, Price LH et al (1998). Tryptophan depletion during continuous CSF sampling in healthy human subjects. Neuropsychopharmacology 19: 26-35.

Chaouloff F (1993). Physiopharmacological interactions between stress hormones and central serotonergic systems. Brain Res Brain Res Rev 18: 1-32.

Cowen PJ (1998). Neuroendocrine challenge tests: what can we learn from them? In: Van de Kar LD (eds). Methods in Neuroendocrinology. CRC Press: Boca Raton, FL, pp 205-223.

De Bellis MD, Geracioti Jr TD, Altemus M, Kling MA (1993). Cerebrospinal fluid monoamine metabolites in fluoxetinetreated patients with major depression and in healthy volunteers. Biol Psychiatry 33: 636-641.

Faull KF, Kraemer HC, Barchas JD, Berger PA (1981). Clinical application of the probenecid test for measurement of monoamine turnover in the CNS. Biol Psychiatry 16: 879-899.

Fischman MW, Foltin RW, Nestadt G, Pearlson GD (1990). Effects of desipramine maintenance on cocaine self-administration by humans. J Pharmacol Exp Ther 253: 760-770.

Fuller RW (1992). The involvement of serotonin in regulation of pituitary-adrenocortical function. Front Neuroendocrinol 13: 250-270.

Fuller RW (1994). Uptake inhibitors increase extracellular serotonin concentration measured by brain microdialysis. Life Sci 55: 163-167.

Geracioti Jr TD, Nicholson WE, Orth DN, Ekhator NN, Loosen PT (1993). Cholecystokinin in human cerebrospinal fluid: concen- trations, dynamics, molecular forms and relationship to fasting and feeding in health, depression and alcoholism. Brain Res 629: 260-268.

Geracioti Jr TD, Orth DN, Ekhator NN, Blumenkopf B, Loosen PT (1992). Serial cerebrospinal fluid corticotropin-releasing hormone concentrations in healthy and depressed humans. J Clin Endocrinol Metab 74: 1325-1330.

Haddock RE, Johnson AM, Langley PE (1989). Metabolic pathway of paroxetine in animals and man and thre comparative pharmacological properties of its metabolites. Acta Psychiatr Scand 80(Suppl 350): 24-26.

Hsiao JK, Agren H, Bartko JJ, Rudorfer MV, Linnoila M, Potter WZ (1987). Monoamine neurotransmitter interactions and the prediction of antidepressant response. Arch Gen Psychiatry 44: 1078-1083.

Invernizzi R, Belli S, Samanin R (1992). Citalopram's ability to increase the extracellular concentrations of serotonin in the dorsal raphe prevents the drug's effect in the frontal cortex. Brain Res 584: 322-324.

Invernizzi R, Bramante M, Samanin R (1996). Role of 5-HT1A receptors in the effects of acute chronic fluoxetine on extracellular serotonin in the frontal cortex. Pharmacol Biochem Behav 54: 143-147.

Jibson M, Faull KF, Csernansky JG (1990). Intercorrelations among monoamine metabolite concentrations in human lumbar CSF are not due to a shared acid transport system. Biol Psychiatry 28: 595-602.

Kirwin PD, Anderson GM, Chappell PB, Saberski L, Leckman JF, Geracioti TD et al (1997). Assessment of diurnal variation of cerebrospinal fluid tryptophan and 5-hydroxyindoleacetic acid in healthy human females. Life Sci 60: 899-907.

Kreiss DS, Lucki I (1994). Differential regulation of serotonin (5$\mathrm{HT}$ ) release in the striatum and hippocampus by 5-HT1A autoreceptors of the dorsal and median raphe nuclei. $J$ Pharmacol Exp Ther 269: 1268-1279.

Leckman JF, Goodman WK, Anderson GM, Riddle MA, Chappell PB, McSwiggan-Hardin MT et al (1995). Cerebrospinal fluid biogenic amines in obsessive compulsive disorder, Tourette's syndrome, and healthy controls. Neuropsychopharmacology 12: 73-86.

Leckman JF, Riddle MA, Berrettini WH, Anderson GM, Hardin M, Chappell $\mathrm{P}$ et al (1988). Elevated CSF dynorphin A [1-8] in Tourette's syndrome. Life Sci 43: 2015-2023.

Leonard BE (1992). Pharmacological differences of serotonin reuptake inhibitors and possible clinical relevance. Drugs 43(Suppl 2): 3-10.

Lucki I (1997). The forced swimming test as a model for core and component behavioral effects of antidepressant drugs. Behav Pharmacol 8: 523-532.

Martensson B, Wagner A, Beck O, Brodin K, Montero D, Asberg M (1991). Effects of clomipramine treatment on cerebrospinal fluid monoamine metabolites and platelet $3 \mathrm{H}$-imipramine binding and serotonin uptake and concentration in major depressive disorder. Acta Psychiatr Scand 83: 125-133.

McCormack HM, Horne DJ, Sheather S (1988). Clinical applications of visual analogue scales: a critical review. Psychol Med 18: $1007-1019$.

McEwen BS (1987). Glucocorticoid-biogenic amine interactions in relation to mood and behavior. Biochem Pharmacol 36: 17551763.

McNair DM, Lorr M, Droppleman LF (1992). EdITS Manual for the Profile of Mood States. Revised 1992. Educational and Industrial Testing Service: San Diego.

Pittoni G, Toffoletto F, Calcarella G, Zanette G, Giron GP (1995). Spinal anesthesia in outpatient knee surgery: 22-gauge versus 25 gauge Sprotte needle. Anesth Analg 81: 73-79.

Potter WZ, Scheinin M, Golden RN, Rudorfer MV, Cowdry RW, Calil HM (1985). Selective antidepressants and cerebrospinal 
fluid. Lack of specificity on norepinephrine and serotonin metabolites. Arch Gen Psychiatry 42: 1171-1177.

Raap DK, Van de Kar LD (1999). Minireview: selective serotonin reuptake inhibitors and neuroendocrine function. Life Sci 65: 1217-1235.

Rabkin JG, Markowitz JS, Ocepek-Welikson K, Wager SS (1992). General versus systematic inquiry about emergent clinical events with SAFTEE: implications for clinical research. J Clin Psychopharmacol 12: 3-10.

Reist C, Helmeste D, Albers L, Chhay H, Tang SW (1996). Serotonin indices and impulsivity in normal volunteers. Psychiatry Res 60: 177-184.

Risby ED, Hsiao JK, Sunderland T, Agren H, Rudorfer MV, Potter WZ (1987). The effects of antidepressants on the cerebrospinal fluid homovanillic acid/5-hydroxyindoleacetic acid ratio. Clin Pharmacol Ther 42: 547-554.

Rutter JJ, Gundlah C, Auerbach SB (1994). Increase in extracellular serotonin produced by uptake inhibitors is enhanced after chronic treatment with fluoxetine. Neurosci Lett 171: 183-186.
LL Carpenter et al

Scheinin M (1985). Monoamine metabolites in human cerebrospinal fluid: indicators of neuronal activity? Med Biol 63: 1-17.

Sheline Y, Bardgett ME, Csernansky JG (1997). Correlated reductions in cerebrospinal fluid 5-HIAA and MHPG concentrations after treatment with selective serotonin reuptake inhibitors. J Clin Psychopharmacol 17: 11-14.

Smith TD, Kuczenski R, George-Friedman K, Malley JD, Foote SL (2000). In vivo microdialysis assessment of extracellular serotonin and dopamine levels in awake monkeys during sustained fluoxetine administration. Synapse 38: 460-470.

Sprotte G, Schedel R, Pajunk H (1987). An 'atraumatic' universal needle for single-shot regional anesthesia: clinical results and a 6 year trial in over 30,000 regional anesthesias. Reg Anaesth 10: 104-108.

Vythilingam M, Anderson GM, Owens MJ, Halaszynski TM, Bremner JD, Carpenter LL et al (2000). Cerebrospinal fluid corticotropin-releasing hormone in healthy humans: effects of yohimbine and naloxone. J Clin Endocrinol Metab 85: 41384145 . 